\title{
IR Camera based system for high speed granulometry measurement on liquid dispersions
}

\author{
by R. Gilblas*, Y. Le Maoult* and N. Lecysyn** \\ * Institut Clément Ader (ICA), Université de Toulouse, CNRS, IMT Mines Albi, UPS, INSA, ISAE-SUPAERO, \\ Toulouse, France, rgilblas@mines-albi.fr \\ ${ }^{* *}$ CEA, DAM, CEA-GRAMAT, F-46500 Gramat, France
}

\begin{abstract}
Liquid dispersions by explosion are still partially unexplored because of the brevity and the complexity of the physical phenomena involved. The theoretical models developed need experimental data as inputs or as validation cases. This work presents the development of a high frequency full-field granulometry method based on the measurement of the transmission in the infrared spectral band. The granulometry is obtained inverting a simple Beer-Lambert's law for each time of the explosion. This paper recalls the theoretical background about optical scattering and presents the experimental set up.
\end{abstract}

\section{Introduction}

The droplets involved in a liquid dispersion by explosion generate a dense cloud traveling at high speed and which granulometry varies with space and time. Few information can be gathered in the literature about the phenomenology and its kinetics. Reference [1] shows that primary liquid fragments arise from instabilities at the liquid/gas interface. This interface expands and form a liquid shell [2]. The atomisation process is due to shearing stresses superior to the superficial tension [3]. A criteria based on a critical Weber number [4] allows then to determine secondary droplets size, whereas a log-normal law [5] describes the droplets diameter distribution. However, experimental results are essential to validate those theories. Some granulometry measurement methods exist and are commercially available [6], but are mainly dedicated to stable and homogeneous dispersions, with low droplets velocities. Deploying new granulometry diagnosis methods at high frequency is then still a major issue for a better understanding of the mecanisms involved in a liquid dispersion growing by explosion. In this work, the method is based on an infrared camera, which measures on-line the extinction coefficient of the liquid dispersion. The first part recalls the theoretical basis of the method and the second part focuses on the experimental set-up.

\section{Theoretical background}

This section presents the link between the optical transmission and the granulometry of a scattering medium. The transmission, under the hypothesis of independent scattering, can be written with the well-known Beer-Lambert's law, recalled hereafter:

$$
\tau(\lambda)=\exp \left(-k_{\text {ext }}(\lambda) * L\right)
$$

With $\lambda$ : wavelength $[\mathrm{m}], \tau$ : transmission, $\mathrm{k}_{\text {ext }}$ : spectral extinction coefficient $\left[\mathrm{m}^{-1}\right] ; \mathrm{L}:$ medium thickness $[\mathrm{m}]$. [7]:

In the case of a heterogeneous medium and for spherical scatterers, the extinction can be predicted by Mie theory

$$
k_{\text {ext }}(\lambda)=\sum_{i=1}^{N} C_{i} \pi r_{i}^{2} Q_{i}\left(\mathrm{n}, \kappa, \lambda, r_{i}\right)
$$

With $\mathrm{N}$ : number of particle classes; $\mathrm{C}_{\mathrm{i}}$ : number of particles by volume for each class (concentration) $\left[\mathrm{m}^{-3}\right]$; $\mathrm{Q}_{\mathrm{i}}$ : efficiency function; $n+i k$ : complex refractive index of the medium.

The efficiency function Qi depends on the refractive index, the scatterer particle size and the wavelength. For large particles $(2 r>>\lambda)$, the efficiency function tends to 2 , whereas for small ones $(2 r<<\lambda)$ it tends to 0 .

For this preliminary study, and to build a simple, fast and full-field granulometry method, a mono-dispersed medium hypothesis will be chosen. An effective radius $r_{m}$ is then introduced and the transmission can then be written as:

$$
\tau=\exp \left(-C \pi r_{m}^{2} Q L\right)
$$


The approach adopted to solve this equation consists in coupling an infrared detector to measure the in-line transmission and two visible fast cameras to verify the geometrical characteristics of the cloud. IR transmission can be measured easily, as it is the ratio of the intensity in presence of the cloud over the intensity without cloud. The concentration $\mathrm{C}$ is considered as constant and is inferred from the total volume of the explosion (the initial volume of liquid is known). The cloud thickness $L$ is measured on line with a visible fast camera located on the top of the experiment.

\section{Experimental set-up}

The cloud is generated by the explosion of a water container (constant volume), with in its centre a given volume of explosive product, which is varying for each experiment. In this way, the energy released by the explosion and the granulometry of the cloud is varying.

The experimental set-up (see figure 1.a) is composed of an infrared camera FLIR SC7000 operating in the spectral band II $B_{\Delta \lambda}=[3-5 \mu \mathrm{m}]$. It can reach acquisition rates up to $1 \mathrm{kHz}$ maximum, which is an important aspect for the dynamical measurement. The infrared source is a flat black body offering an emitting surface of $15^{*} 15 \mathrm{~cm}^{2}$ (homogeneous high emissivity of 0.95 ), and a maximum temperature of $500^{\circ} \mathrm{C}$. Between those two elements is deployed the high speed water cloud, creating the variation of transmission versus time. On the top of the cloud is placed a first PHANTOM high speed $(5 \mathrm{kHz})$ camera, measuring the thickness of the cloud, and another one is placed remotely, in the axis of the container, to measure the radius of the explosion (see figure.1.b).

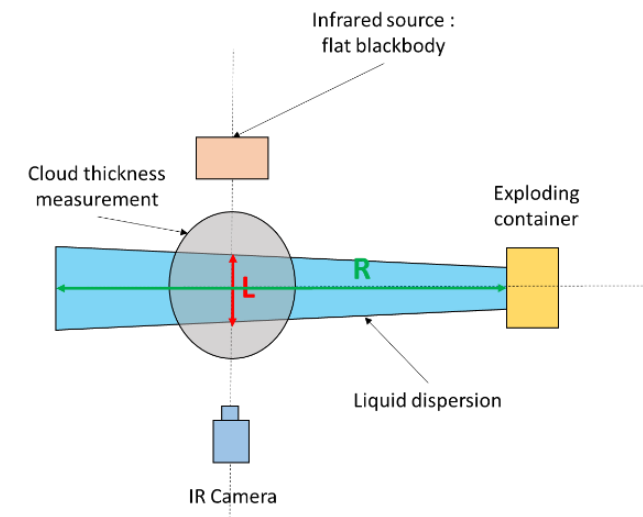

a)

Visible camera for the measurement of the
explosion radius

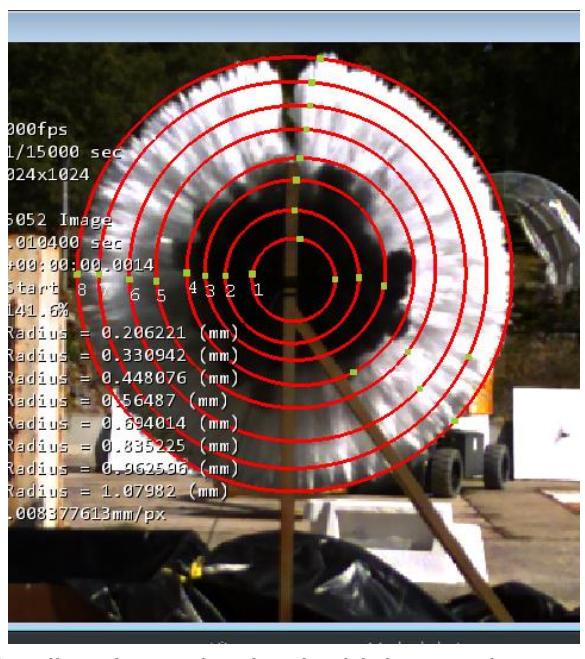

b)

The results will be discussed in details in the final article. A specific study will treat about the selection of the relevant time where the majority of the liquid is dispersed. Then a mean concentration will be calculated and injected in the transmission equation (Equ.3). The measurement of the cloud thickness (and its possible extrapolation) will also feed this equation.

To conclude, this method enables to qualify the different domains of the explosion, so it leads to a better understanding of the phenomenology of the explosion. Moreover, it showed good robustness characteristics and the results are in fair agreement with the simulation and previous campaigns. The extinction method give a good first estimation of the droplets mean radius composing the high speed cloud.

\section{REFERENCES}

[1] Lei, Y. \& al. (2016). Experimental study of axisymmetrical dissemination of liquid driven by shockwave. Journal of experiments in fluid mechanics, 30(6), 32-37.

[2] Murray, S \& al.,(2009). Spray detonation (Vol. ShockWave Science and Technology Reference Library, vol. 4). Medicine Hat, Canada: Springer.

[3] Pilch, M. \& al.,(1987). Use of breakup time data and velocity history data to predict the maximum size of stable fragments for acceleration-induced breakup of a liquid drop. Int. J. Multiphase Flow, 13(6),741-757.

[4] Shi, H. \& al. (2009) Experimental study on the mechanism of the Richtmyer-Meshkov instability at a gas-liquid interface. Journal of Hydrodynamics, 21(3), 423,428.

[5] Singh, S. \& al. (2001). Extended near-field modelling and droplet size distribution for fuel-air explosive warhead. Defence Science Journal, 51(3), 303-314.

[6] A.Coghe \& al., "Quantitative Optical Techniques for denses sprays investigation ; a survey », Optics and lasers in engineering, 50 (2012), 46-56.

[7] H.C van de Hulst, « Light scattering by small particles », Dover ed. ,1981 